Supporting Information for:

\title{
Thermal field-flow fractionation for characterization of architecture in hyperbranched aromatic-aliphatic polyesters with controlled branching
}

\author{
William C. Smith, ${ }^{\dagger}$ Martin Geisler, ${ }^{\ddagger}, \S$ Albena Lederer, ${ }^{\ddagger}$, , S. Kim Ratanathanawongs Williams, ${ }^{*}, \dagger$ \\ 'Department of Chemistry, Colorado School of Mines, Golden, USA \\ Leibniz-Institut für Polymerforschung Dresden e.V., Germany \\ ${ }^{\S}$ School of Science, Faculty of Chemistry and Food Chemistry, Technische Universität Dresden, 01062 \\ Dresden, Germany
}

Table of content for the supporting information:

1. Increment of Refraction $(d n / d c)$ Determination $\quad S-2$

2. Temperature Programmed Dynamic Light Scattering S-3

3. Online ThFFF-MAL-DLS ( $R_{h}$ versus $M_{w}$ Conformation Plots Data and Fits) S-5

4. Molecular Weight versus Soret Calibration using ThFFF-MALS S-6

5. Polyester Room Temperature SEC $\quad$ S-7

6. Isolation and Characterization of Cyclic Polyester Subpopulations $\quad$ S-8

7. Thermal FFF Multiangle Light Scattering Overlays S-10

8. References S-11 


\section{Determination of Specific Refractive Index Increment for Polyester Samples}
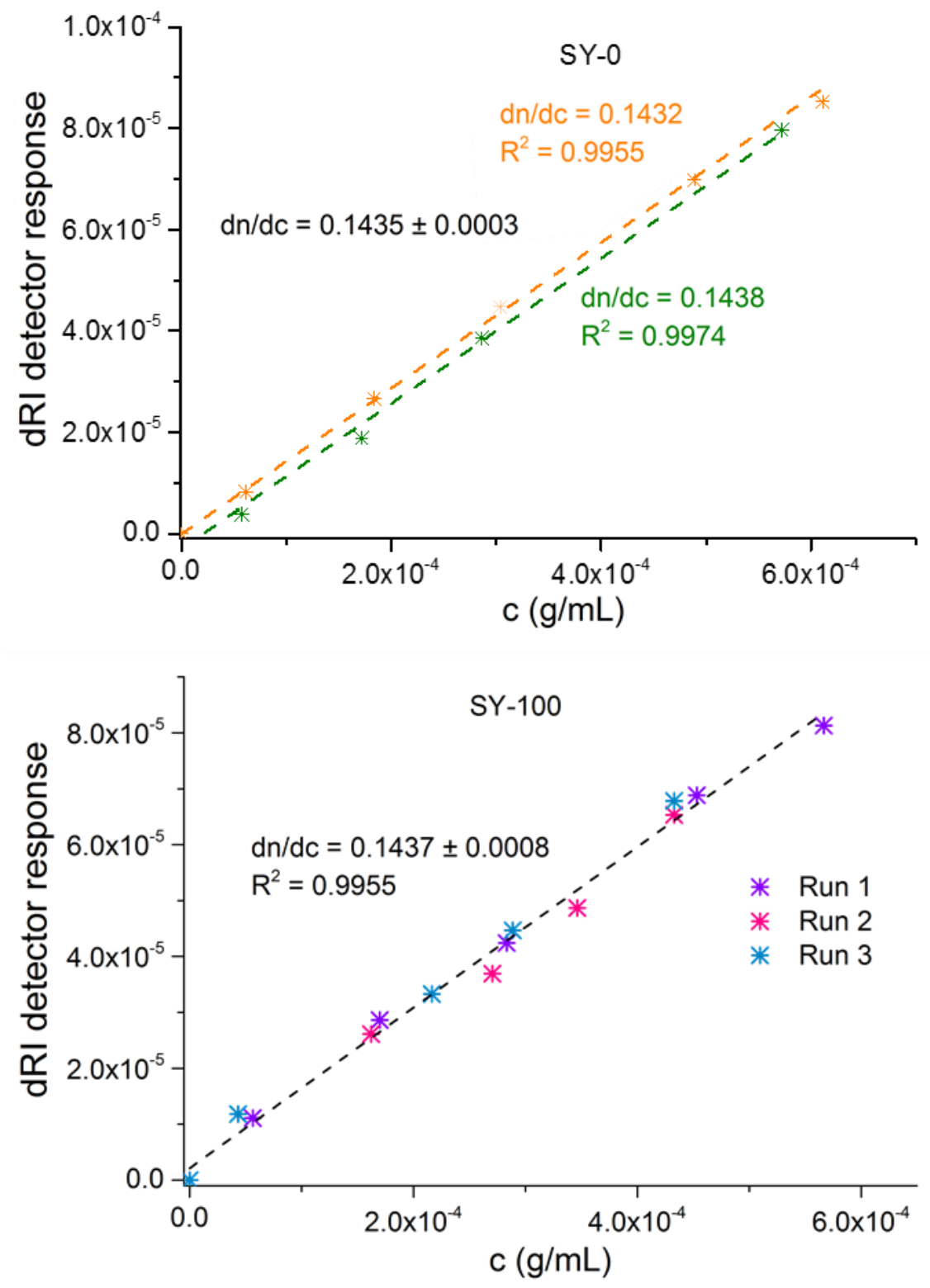

Figure S1. Representative data for determination of increments of refraction $(d n / d c)$ for linear $(\mathrm{SY}-0)$ and pseudodendrimer (SY-100) polyester in cyclohexane. SY- 0 and SY-100 exhibit the same $d n / d c$ within experimental error. The $\mathrm{dn} / \mathrm{dc}$ is independent of branching for these aliphatic-aromatic polyesters. An average $d n / d c$ value of $0.1435 \mathrm{~mL} / \mathrm{g}$ was used in subsequent analyses in cyclohexane. 


\section{Temperature Programmed Batch-Mode Dynamic Light Scattering}

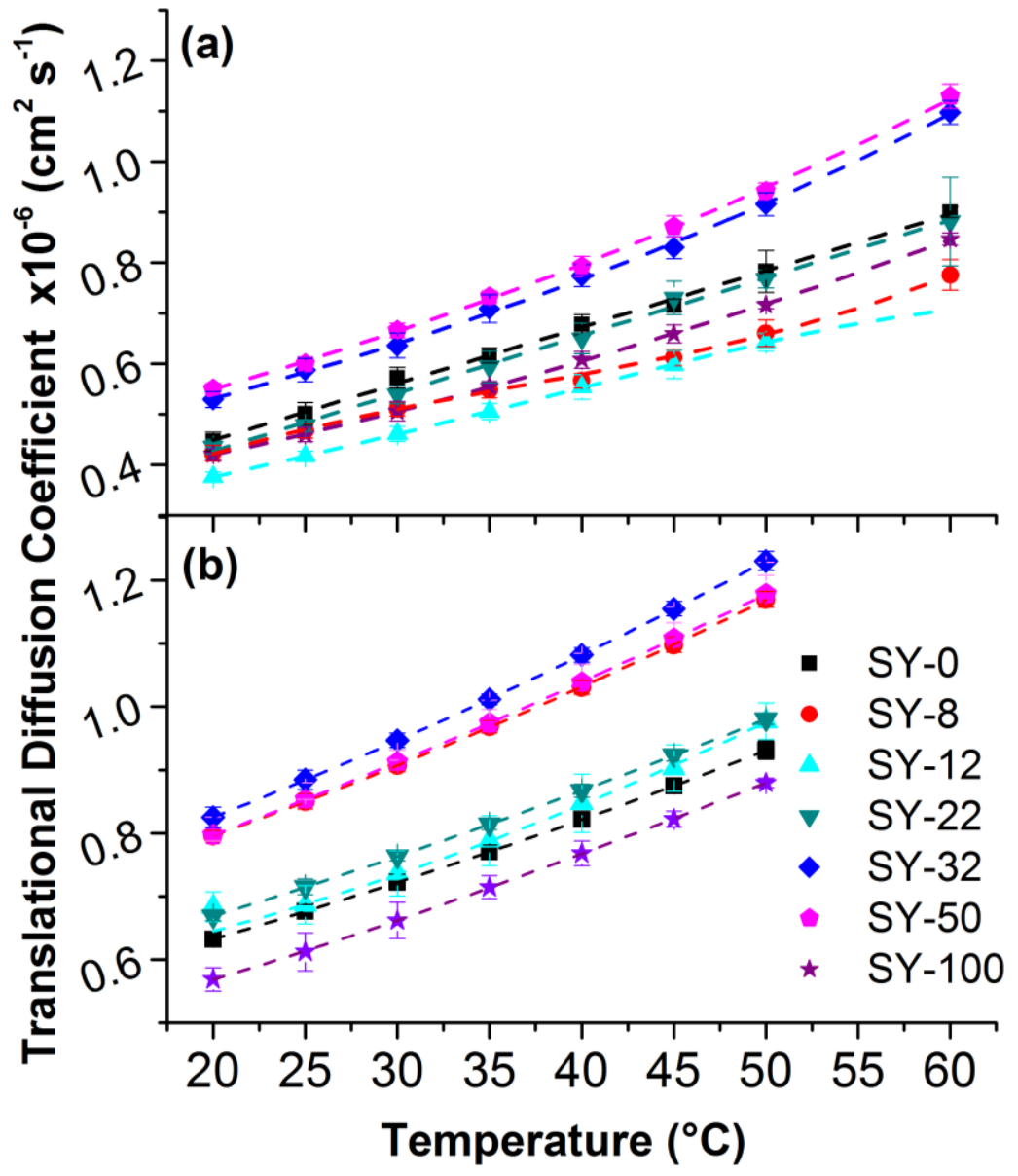

Figure S2. Translational diffusion coefficients versus temperature for all DB Polyester samples in a) cyclohexane and b) tetrahydrofuran. Regression lines have been added for ease in examining trends with respect to temperature. For the calculation of $D_{T}$ from ThFFF experiments at high temperature $(\Delta T \geq 65 \mathrm{~K})$ the values of $D$ used must be determined based on the temperature at the mean layer thickness (or height in the channel, ' $l$ ') from the average sample retention time $\left(t_{r}\right)$. Values of $D$ for all samples are listed in Tables 2 . For all samples in both solvents, the temperature at the mean layer thickness was between $36-40{ }^{\circ} \mathrm{C}$. Determining the temperature profile in the ThFFF channel is covered by Belgaied et al. ${ }^{1}$ Mean layer thickness is related to $t_{r}$ and void time $t^{0}$ via Equation (S1):

$R=\frac{t^{0}}{t_{r}}=6 \lambda\{v+(1-6 \lambda v)[\operatorname{coth}(1 / 2 \lambda)-2 \lambda]\}$

where the retention parameter $\lambda$ is a ratio between ' $l$ ' and the channel's thickness $(w)$. The $\lambda$ is also directly related to the physicochemical properties of the analyte based on the field driven transport (thermal diffusion ' $D_{T}$ ' in ThFFF), diffusional transport $(D)$, and the magnitude of the field (temperature difference across the channel ' $\Delta T$ ') as given by Equation (S2):

$\lambda=\left(S_{T} \Delta T\right)^{-1}=\frac{D}{D_{T} \Delta T}$

Equation (S1) is an adjusted form of the generalized FFF retention equation. The factor $(v)$ is used to correct for distortion of the parabolic flow based on the temperature dependence of the solvent viscosity. Values for $v$ can be found in existing literature. ${ }^{2}$ 


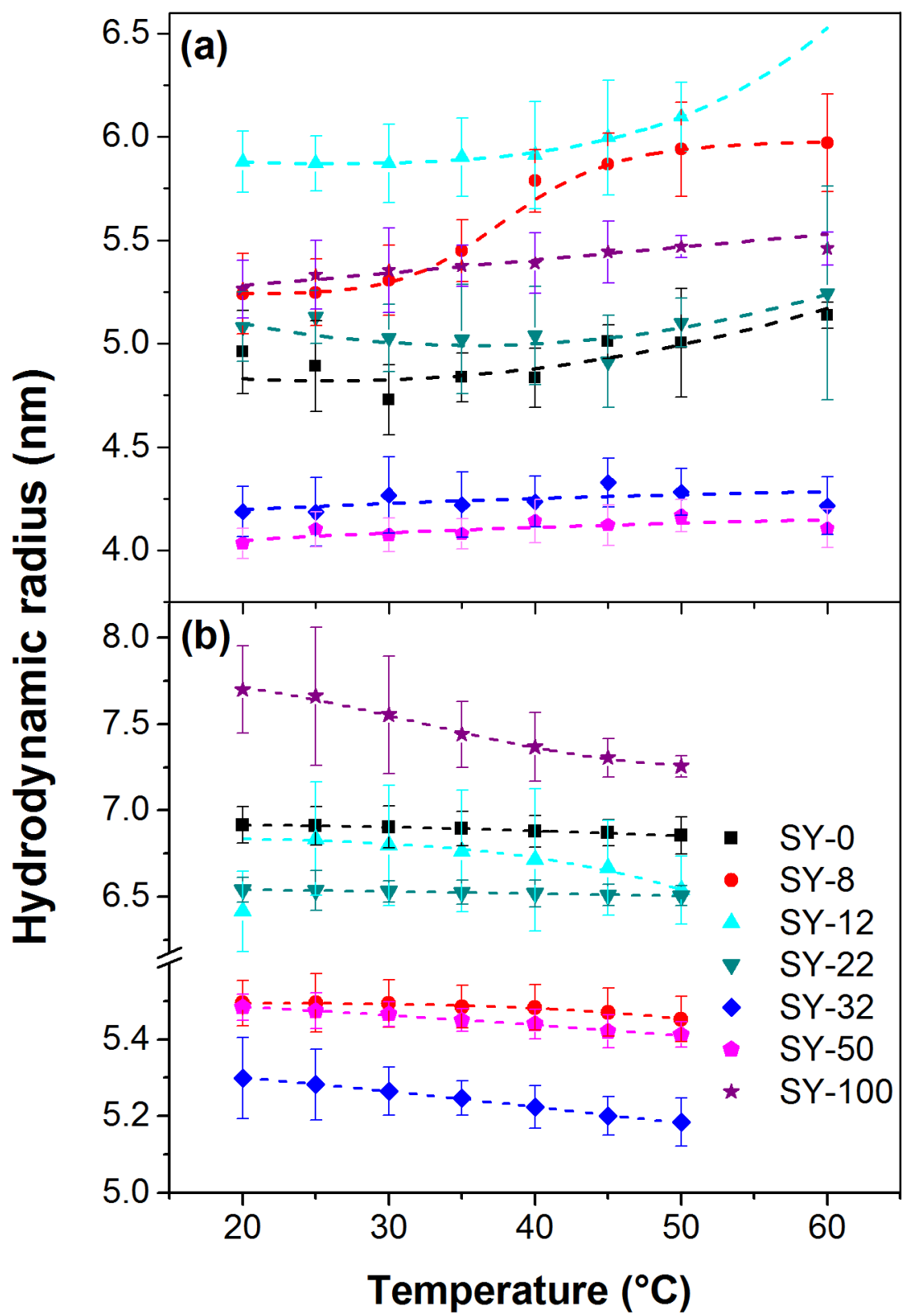

Figure S3. Temperature dependence of hydrodynamic radius $\left(R_{h}\right)$ for all DB polyester samples in a) cyclohexane and b) tetrahydrofuran. Regression lines have been added for ease in examining trends with respect to temperature. Error bars represent measurement standard deviations. 


\section{3. $R_{h}$ versus $M_{w}$ Conformation Plots Data and Fits}

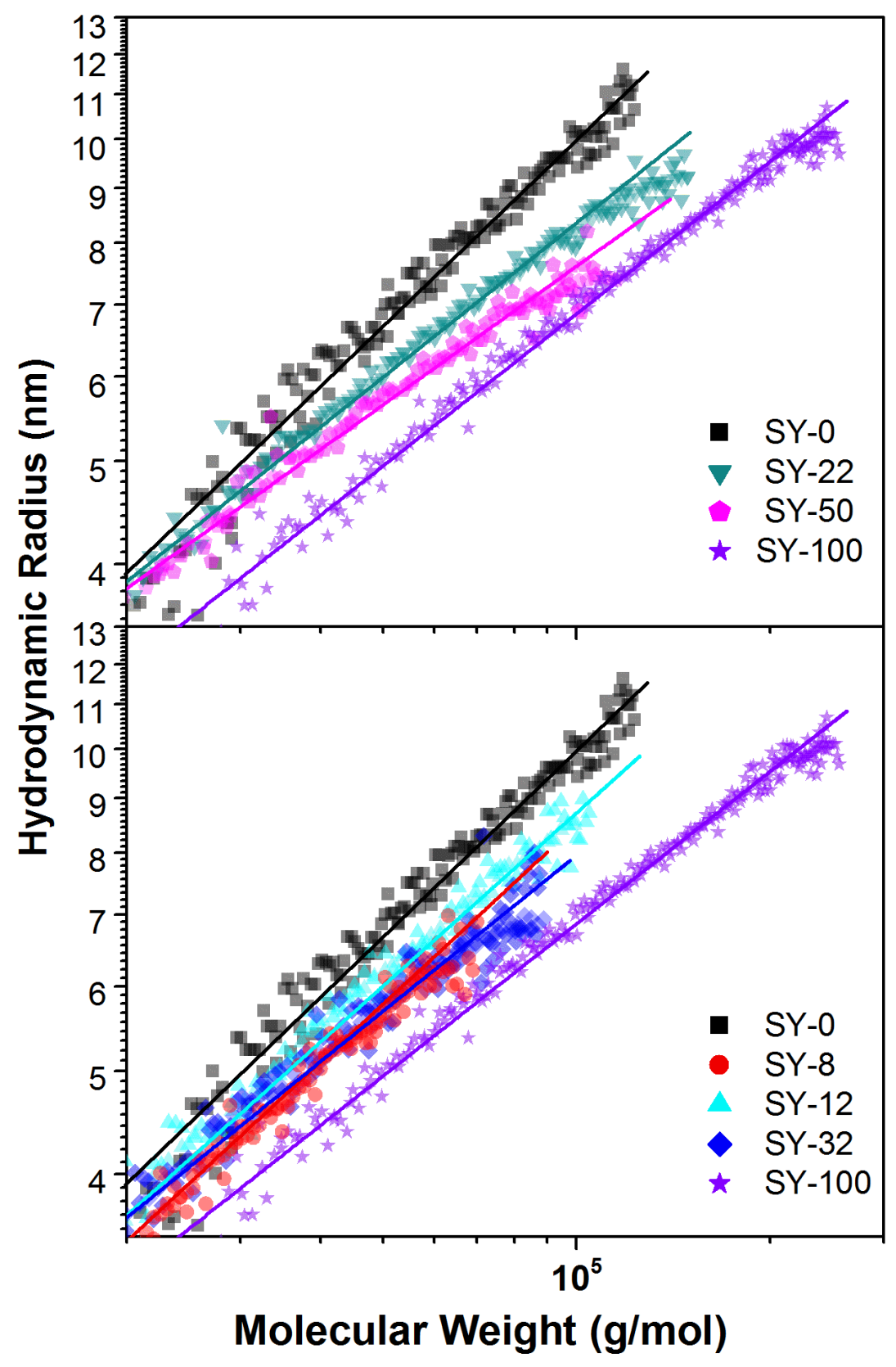

Figure S4. Conformation plots from ThFFF-DLS-MALS for all samples in THF. The allometric fits (solid lines) are overlaid on $R_{h}$ and $M_{w}$ (symbols) from DLS and MALS. Scaling parameter values can be found in Figure $3 \mathrm{~b}$ and $\mathrm{c}$.

Fractionation was performed using a TF2000 model ThFFF channel (Postnova Analytics, Salt Lake City, UT, USA) coupled online to a Dawn Heleos II multiangle light scattering (MALS)-dynamic light scattering (DLS) detector (Wyatt Technology Corporation, Santa Barbara, CA, USA), and an Optilab T-rEX differential refractive index detector (Wyatt Technology Corporation, Santa Barbara, CA). The ThFFF channel was made from a $127 \mu \mathrm{m}$ polyimide spacer with a ribbon-like section removed that was $2.0 \mathrm{~cm}$ in breadth and $45.6 \mathrm{~cm}$ in length from inlet to outlet. The carrier liquid was tetrahydrofuran (THF) pumped through the channel at $0.2 \mathrm{~mL} / \mathrm{min}$. A $20 \mu \mathrm{L}$ sample loop was used to introduce the sample into the channel. For all experiments, the cold wall, MALS-DLS, and dRI flow cells were maintained at $30 \pm 1{ }^{\circ} \mathrm{C}$. DLS data was collected with 2 second acquisition times. 


\section{Soret Coefficient versus Molecular Weight Calibration using ThFFF-MALS}

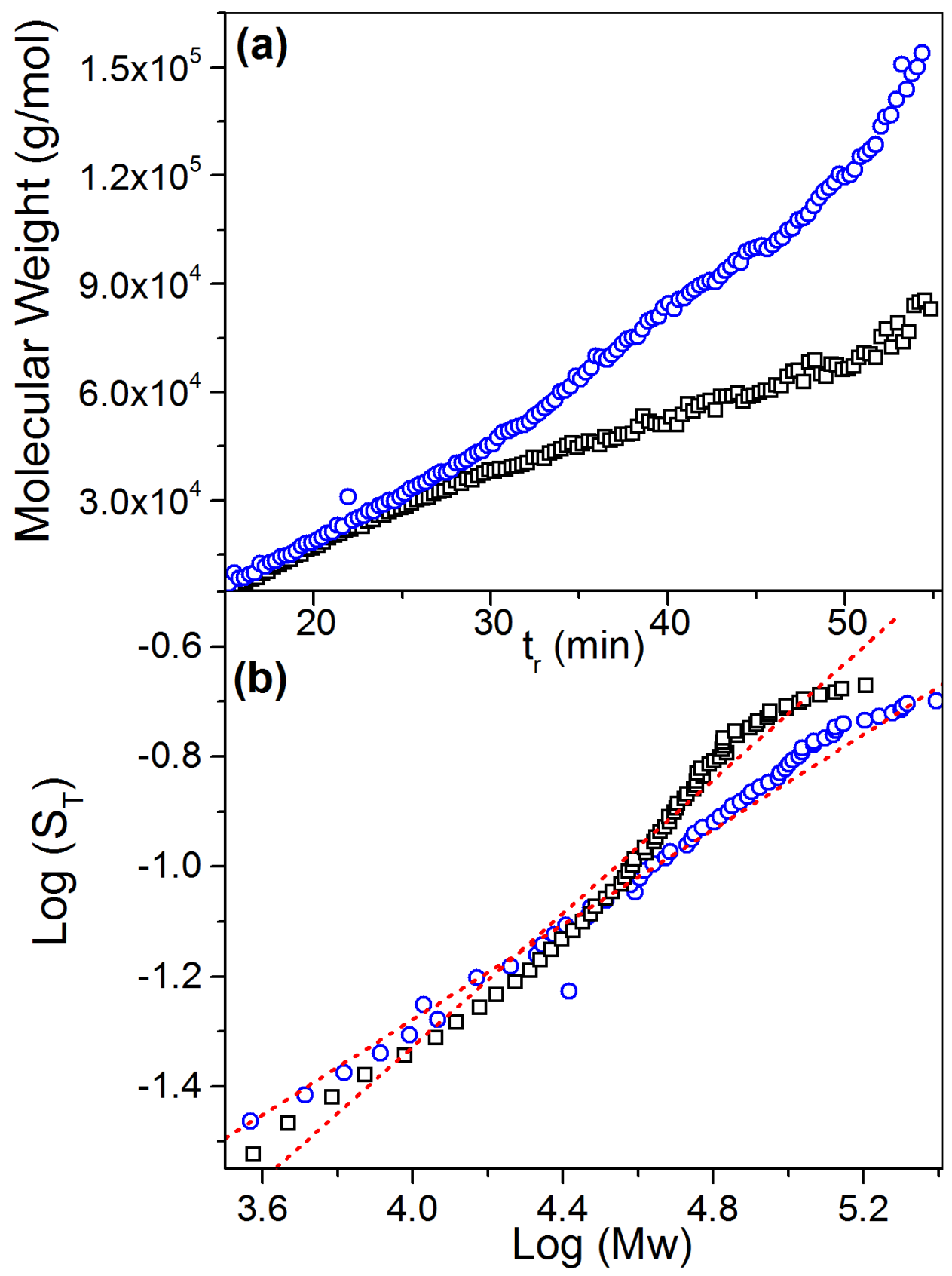

Figure S5. a) Molecular weight versus retention time for the linear SY-0 polyester in cyclohexane (black) and tetrahydrofuran (blue) at $\Delta T: \sim 100{ }^{\circ} \mathrm{C}$ and $T_{c}: \sim 23{ }^{\circ} \mathrm{C}$ for both solvent systems. For the same retention time, $M w$ and $S_{T}$ are higher in THF. However, at equivalent $M_{w}$ both retention time and $S_{T}$ are higher in cyclohexane. This indicates that the $M_{w}$-based selectivity is higher in cyclohexane than THF. b) Molecular weight calibration with the linear SY-0 polyester in cyclohexane (black) and tetrahydrofuran (blue) at $\Delta T: \sim 100{ }^{\circ} \mathrm{C}$ and $T_{c}: \sim 23{ }^{\circ} \mathrm{C}$ for both solvent systems. Soret coefficients are calculated using Equation (S1)-(S2) and compared to $M_{w}$ from MALS at the corresponding retention times. Correlation of $\log \left(M_{w}\right)$ and $\log \left(S_{T}\right)$ allows prediction of the Soret coefficient of a linear analogue for use in Equation (2) of the main article to produce $g$ " values at a given measured molecular weight for each branched sample. 


\section{Polymer Size Exclusion Chromatography with MALS and dRI}

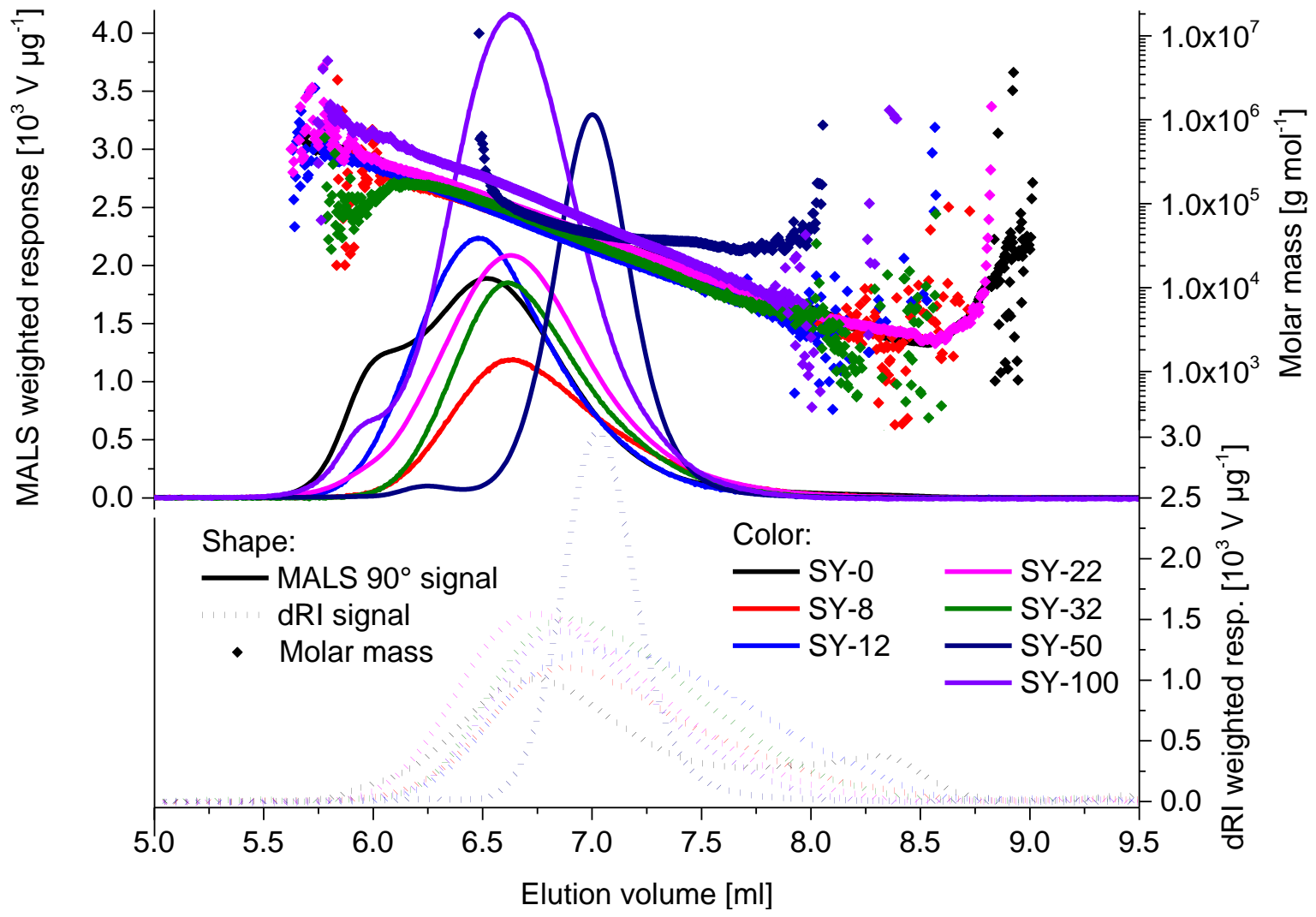

Figure S6. Overlay of SEC-MALS fractograms of linear (SY-0), gradually branched (SY-8, SY-12, SY-22, SY32), hyperbranched (SY-50), and pseudodendrimeric (SY-100) polyesters from Figure 5 in the main article. Solid lines for MALS response and $M w$ (top half), dotted lines for dRI response (bottom half), both weighted by the detected mass. Presence of a low molecular weight subpopulation in the SY-0 can be seen at $8.3 \mathrm{~mL}$.

The determination of the molecular weights given in Table 1 were performed by SEC-MALS using a flow rate of $1 \mathrm{~mL} / \mathrm{min}$. The SEC was equipped with an HPLC system (pump, degasser and manual control unit, Series 1200 by Agilent Technologies Inc., Santa Clara, CA, USA), a manual injection valve with a fixed injection volume of $53 \mu 1$, a PLgel $5 \mu \mathrm{m}$ Mixed C chromatography column $(300 \mathrm{~mm} \times 7.5 \mathrm{~mm}$, Agilent Technologies Inc.) and was coupled to a viscosity/differential refractive index (dRI) dual detector (ETA-2020, WGE Dr. Bures, Germany) and a multiangle laser light scattering detector (MALS) Tristar® miniDawn (Wyatt Technology Corporation, USA, $\lambda=632 \mathrm{~nm}$ ). The carrier fluid was THF (Acros, Germany) and the analyte concentrations were about 1.5 to $2 \mathrm{mg} / \mathrm{mL}$. All evaluations were done with the software ASTRA 4.9 and 6.1.2 (Wyatt Technology Corporation). The molecular weight calculations were done using the Zimm formalism and $\mathrm{d} n / \mathrm{d} c$ values of $0.1550 \mathrm{~mL} / \mathrm{g}$ as used previously., 


\section{Isolation and Characterization of Cyclic Polyester Subpopulations}

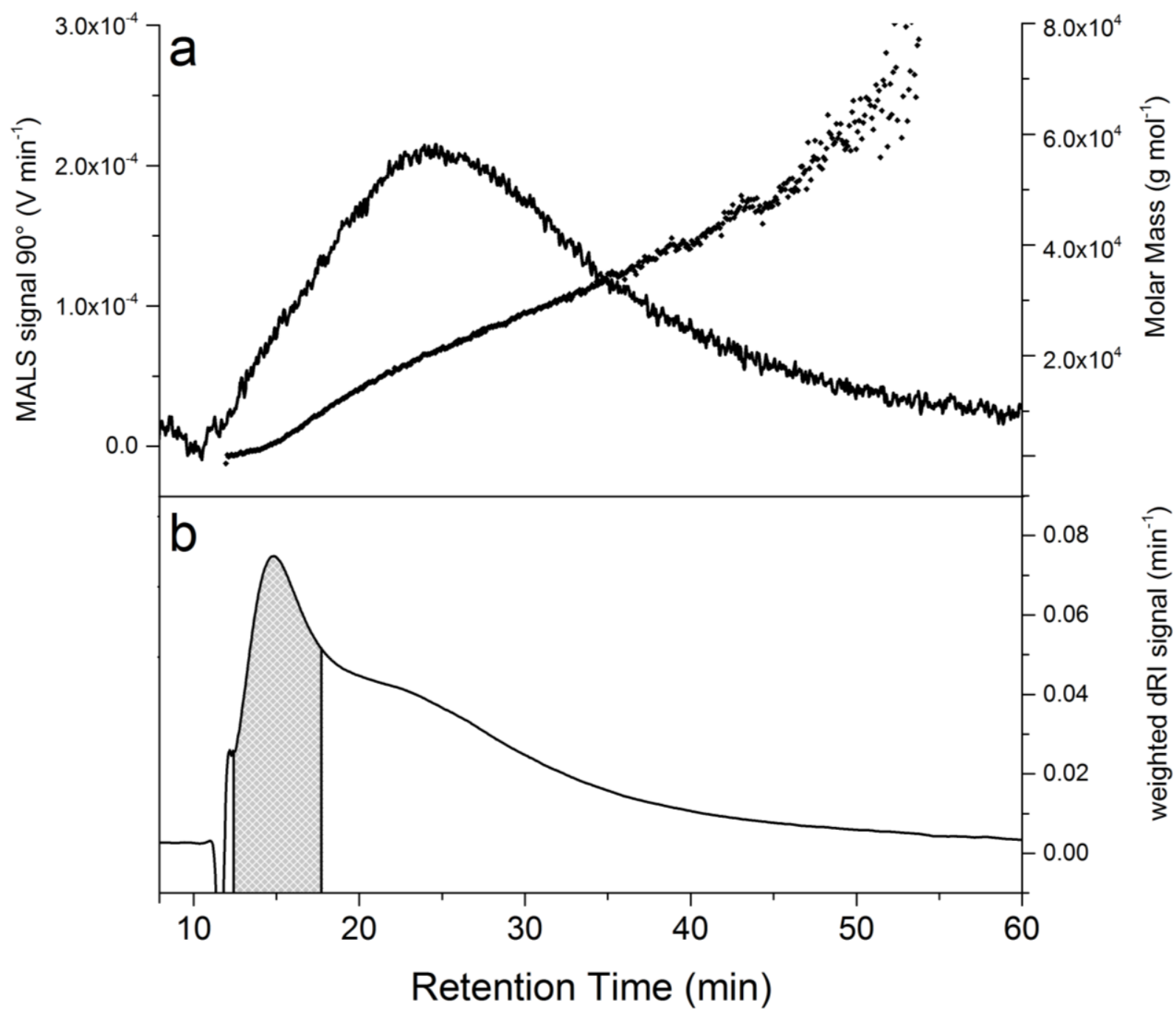

Figure S7. Fractogram of the linear SY-0 polyester in CH with a) MALS detector response and overlaid $M_{w}$ and b) dRI response. Fraction collection interval from 12.5 to 17.5 minutes indicated by the shaded grey region.

\section{Matrix assisted laser desorption time of flight mass spectrometry (MALDI-TOF-MS)}

MALDI-TOF mass spectrometry is used to distinguish cyclized structures from their linear analogues. ${ }^{5}$ Mass spectra were recorded using a Bruker UltrafleXtreme (Bruker Daltronics, Billerica, MA, USA) time-of-flight mass spectrometer. Low molecular weight fractions from SY-0 and SY-8 were prepared following a published

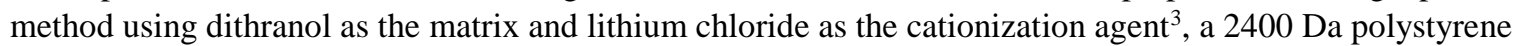
(Polymer Source, Dorval, Quebec, Canada) was used for calibration. The irradiation was provided by a nitrogen pulse laser at $337 \mathrm{~nm}$ with the 'minimum' spot size, pulses at $1000 \mathrm{~Hz}$ and $80 \%$ power. The measurement conditions were as follows: reflectron-mode, positive polarity, $25 \mathrm{kV}$, detector gain at $10 \mathrm{x}$, and pulsed ion extraction at $100 \mathrm{~ns}$. 

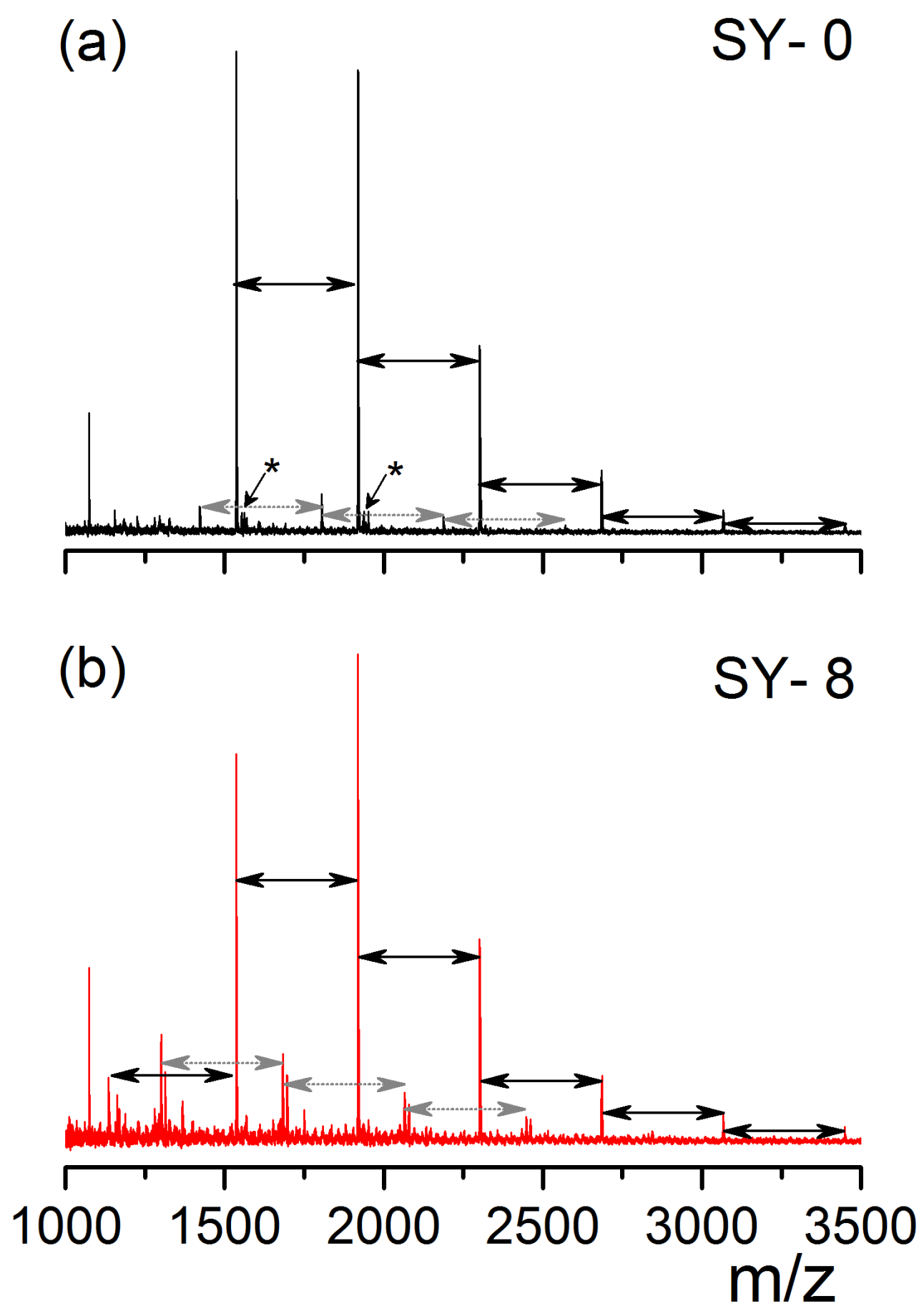

Figure S8. MALDI-TOF MS spectrum of a) SY-0 cyclic ThFFF fraction and b) SY-8 cyclic fractions collected according to the positive g" regions in Figure $5 \mathrm{~b}$. Spacing between peaks (indicated by dual arrows) represents the addition of single monomer units of $382.5 \mathrm{amu}$ (arbitrary mass units). A low intensity population of with identical spacing (dual grey arrows) may be due to fragmentation during fly through the reflectron. The mass difference between the two populations is $\sim 140$ amu and may be due to loss of a tert-butyldimethylsilyl group.

* Indicates the presence of a linear analogue to the polymer cycle at 18 amu greater mass. 


\section{Thermal FFF with Multiangle Light Scattering Overlays}

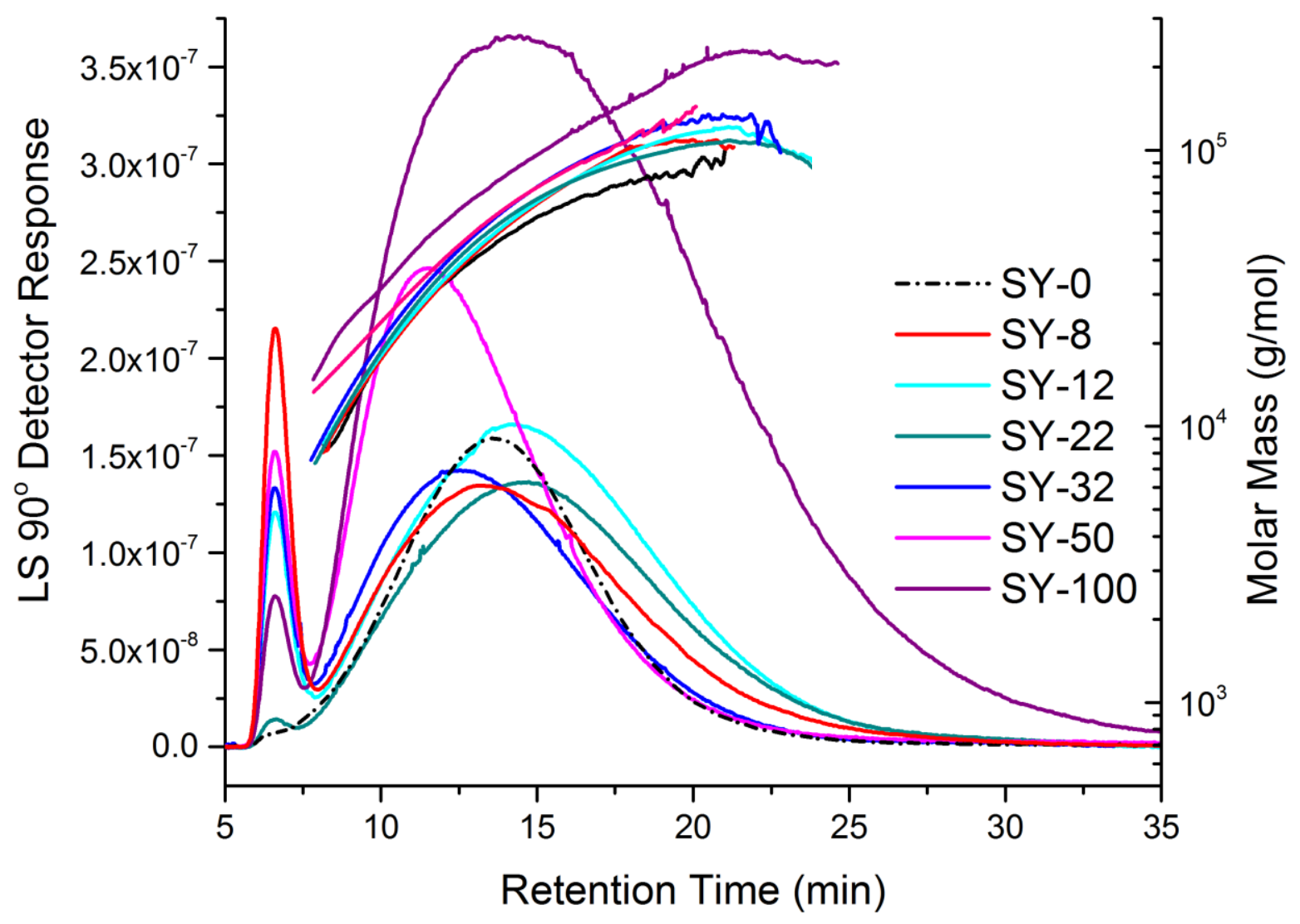

Figure S9. MALS fractograms with overlaid molar mass for all polyester samples in $\mathrm{CH}$. Conditions: $\Delta T: 100{ }^{\circ} \mathrm{C}$, $T_{C}: 30^{\circ} \mathrm{C}$; channel thickness $127 \mu \mathrm{m}$. 


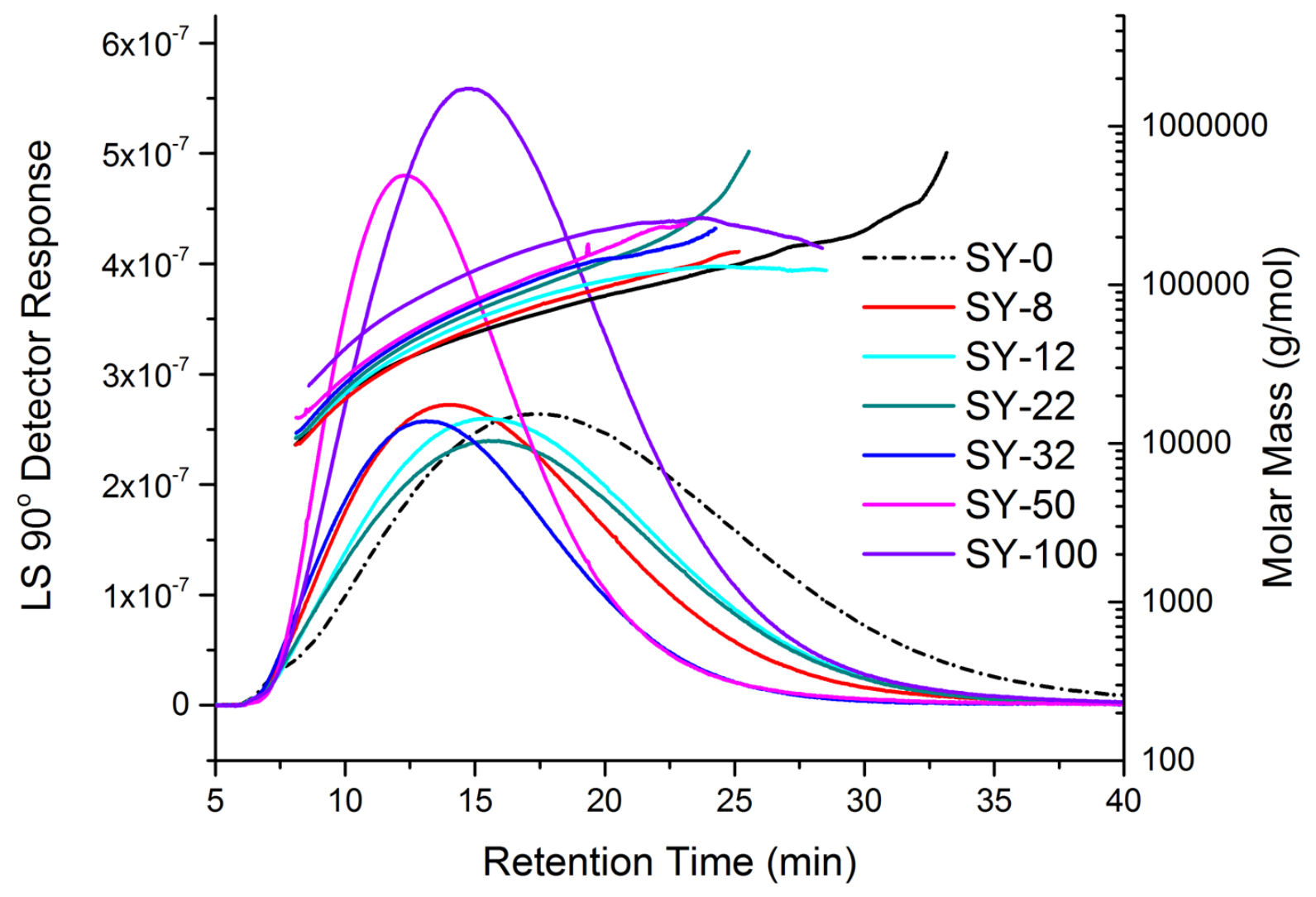

Figure S10. MALS fractograms with overlaid molar mass for all polyester samples in THF. Conditions: $\Delta T: 100{ }^{\circ} \mathrm{C}$, $T_{C}: 30^{\circ} \mathrm{C}$, channel spacer $127 \mu \mathrm{m}$.

\section{References:}

(1) Belgaied, J. E.; Hoyos, M.; Martin, M. Velocity Profiles in Thermal Field-Flow Fractionation. J. Chromatogr. A 1994, 678 (1), 85-96. https://doi.org/10.1016/0021-9673(94)87077-2.

(2) Gunderson, J. J.; Caldwell, K. D.; Giddings, J. C. Influence of Temperature Gradients on Velocity Profiles and Separation Parameters in Thermal Field-Flow Fractionation. Sep. Sci. Technol. 1984, 19 (10), 667-683. https://doi.org/10.1080/01496398408060668.

(3) Khalyavina, A.; Schallausky, F.; Komber, H.; Al Samman, M.; Radke, W.; Lederer, A. Aromatic-Aliphatic Polyesters with Tailored Degree of Branching Based on $\mathrm{AB} / \mathrm{AB}{ }_{2}$ and $\mathrm{ABB} * / \mathrm{AB}_{2}$ Monomers. Macromolecules 2010, 43 (7), 3268-3276. https://doi.org/10.1021/ma100037n.

(4) Lederer, A.; Burchard, W.; Khalyavina, A.; Lindner, P.; Schweins, R. Is the Universal Law Valid for Branched Polymers? Angew. Chemie Int. Ed. 2013, 52 (17), 4659-4663. https://doi.org/10.1002/anie.201209228.

(5) Sepulchre, M.; Sepulchre, M.-O.; Belleney, J. Aliphatic-Aromatic Hyperbranched Polyesters by Polycondensation of Potassium 3,5-Bis(Bromomethyl)Benzoate: Formation of Cyclic Structures. Macromol. Chem. Phys. 2003, 204 (14), 1679-1705. https://doi.org/10.1002/macp.200350023. 